\title{
ERGOTISMO DEL SISTEMA NERVIOSO CENTRAL. REPORTE DE DOS MUERTES MATERNAS ASOCIADAS A VASOESPASMO CEREBRAL POR MEDICAMENTOS DERIVADOS DEL ERGOT EN ANTIOQUIA, COLOMBIA, Y REVISIÓN DE LA LITERATURA
}

\section{Ergotism of the central nervous system. Report of two maternal deaths associated with cerebral vasospasm due to ergot-dlerived drugs in Antioquia, Colombia, and review of the literature}

Jesús A. Velásquez-Penagos, $M D^{1}$; Jorge Mario Gómez-Jiménez, $M D^{2}$; Bernardo Agudelo-Jaramillo, $M^{3}$

Recibido: octubre 22/15 - Aceptado: septiembre 16/16

\section{RESUMEN}

Objetivo: presentar dos casos de muerte materna asociados al uso de medicamentos derivados del ergot (ergotismo agudo severo), y realizar una revisión de la literatura de la presentación de eventos adversos en el sistema nervioso central en puérperas expuestas a estos medicamentos.

Materiales y métodos: se presentan dos casos de muerte materna posparto, el primero se asoció al uso de bromocriptina para supresión de lactancia y el segundo al uso metilergometrina para controlar he-

1 Ginecoobstetra, Universidad de Antioquia; cuidado intensivo obstétrico. Profesor del Departamento de Ginecología y Obstetricia, Universidad de Antioquia; ginecoobstetra, Centro NACER, Universidad de Antioquia; ginecoobstetra Hospital Universitario San Vicente Fundación, Medellín (Colombia).susve@yahoo.com

2 Ginecoobstetra, Universidad de Antioquia. Profesor del Departamento de Ginecología y Obstetricia, Universidad de Antioquia, Medellín (Colombia).

3 Ginecoobstetra, Universidad de Antioquia; epidemiólogo. Profesor del Departamento de Ginecología y Obstetricia, Universidad de Antioquia; ginecoobstetra, Centro NACER, Universidad de Antioquia, Medellín (Colombia). (Colombia). morragia poscesárea. Las pacientes fueron atendidas en instituciones de tercer nivel de complejidad en la ciudad de Medellín, Colombia. Se realizó revisión de la literatura, registrada en la base de datos Medline vía PubMed. Los términos empleados para la búsqueda fueron: derivados del ergot, bromocriptina, angeítis cerebral posparto, ergotismo, enfermedad vascular cerebral posparto. Se buscaron, sin límite de tiempo, reportes de caso, reportes de series de caso y revisiones de tema. Se buscaron informes o alertas de seguridad de agencias reguladoras tales como: la Federal Drugs Administration (FDA), la European Medicines Agency (EMA) y del Instituto Nacional de Vigilancia de Medicamentos y Alimentos (Invima). Se consultaron artículos en inglés, francés y español. Resultados: se incluyeron 16 publicaciones que cumplieron con los criterios de búsqueda. Se lograron identificar 33 casos. Dos fueron fatales, uno asociado al uso de metilergonovina venosa para el alumbramiento y el otro a ergometrina oral usado como abortivo en la semana 20 de gestación. En las 
neuroimágenes predominan los hallazgos isquémicos (sugestivos de vasoespasmo cerebral). En tres casos se reportó hemorragia intracerebral, uno de estos fue un caso fatal. Los síntomas más frecuentes de presentación fueron la cefalea intensa, seguida de la convulsión. Solo en nueve casos se logró identificar el antecedente de hipertensión o preeclampsia, y en cuatro migraña. La indicación para el uso de bromocriptina en todos los casos fue suprimir la lactancia. En los tres casos reportados en que se usó metilergonovina fue para realizar alumbramiento. En el sistema de farmacovigilancia colombiano no se encontraron reportes de eventos adversos serios asociados a estos medicamentos.

Conclusión: se debe reconocer el ergotismo del sistema nervioso central en el puerperio por el uso de medicamentos tales como la bromocriptina y la metilergonovina, como una entidad potencialmente fatal. Es importante crear una cultura de reporte de eventos adversos serios de estos medicamentos en nuestro país.

Palabras clave: mortalidad materna, ergotismo, alcaloides derivados del ergot, vasoespasmo cerebral, posparto.

\section{ABSTRACT}

Objective: To report two cases of maternal death associated with ergot-derived drugs (acute sever ergotism), and to conduct and review of the literature on central nervous system adverse events during the postpartum period in women exposed to these medications.

Materials and Methods: Two cases of maternal death during the postpartum period. The first was associated with the use of bromocriptine for breast milk suppression, and the second was associated with the use of methylergometrine for the control of bleeding after Cesarean section. The patients received care at Level III institutions in the city of Medellín, Colombia. A review of the literature was conducted in the Medline database through Pubmed. The terms used for the search were: ergot derivatives, bromocriptine, postpartum cerebral angiitis, ergotism, postpartum cerebral vascular disease. The search was conducted without a time limitation and included, case reports, case series reports, and reviews. The search also included safety reports or alerts from regulatory agencies such as the FDA, the European Medicines Agency (EMA), and Invima. Articles in English, French and Spanish were reviewed.

Results: Overall, 16 publications that met the search criteria were included, and 33 cases were identified. Two of the cases were fatal, one associated with the use of intravenous methylergonovine for delivery and the second one was associated with the use of oral ergometrine to induce abortion at 20 weeks of gestation. Neuroimaging studies show, predominantly, ischemic findings (suggestive of cerebral vasospasm). In three cases, intracranial haemorrhage was reported, and one of the three cases was fatal. The most frequent presenting symptoms were intense headache, followed by seizures. It was possible to identify a history of hypertension and/or preeclampsia only in nine cases, and a history of migraine in four. The vast majority of patients were otherwise healthy. In all the cases, the indication for using bromocriptine was breast milk suppression. In the three reported cases in which methylergonovine was used, the indication was to assist delivery. No reports of serious adverse events associated with these drugs were found in the Colombian pharmacovigilance system.

Conclusion: Ergotism of the central nervous system due to the use of drugs such as bromocriptine and methylergonovine must be recognised during the postpartum period because it is life-threatening. It is important to create a culture of reporting of serious adverse events associated with these medications in our country.

Key words: Maternal mortality, ergotism, ergotderived alkaloids, cerebral vasospasm, postpartum.

\section{INTRODUCCIÓN}

Las complicaciones neurológicas en el puerperio son eventos poco frecuentes pero de alta letali- 
dad. La causa más frecuente son los trastornos hipertensivos de la gestación (1). Sin embargo, en ciertas circunstancias clínicas se deben considerar diagnósticos diferenciales, tales como el ergotismo del sistema nervioso central, que hace parte de una entidad conocida como angiopatía cerebral posparto (también se conoce como angitis posparto, síndrome de Call-Flemming o síndromes de vasoconstricción cerebral posparto). Se ha relacionado con medicamentos entre los que se encuentran la metilergometrina y la bromocriptina que son de uso frecuente en la práctica ginecológica y obstétrica (2). Los síntomas, por los efectos sobre el sistema nervioso central (SNC), son variados: sequedad de mucosas, alucinaciones, letargia, distorsión motora del tronco y extremidades, alteraciones visuales, cefalea y convulsiones. La mortalidad del ergotismo convulsivo oscila entre el 10-20\% (1). El tiempo de aparición de estos síntomas después de la exposición al fármaco puede variar desde minutos (metilergonovina) a días (bromocriptina) (2).

En términos históricos es importante recordar la epidemia de ergotismo en la Edad Media, conocida como el "fuego de San Antonio", que motivó la aparición de monasterios, especialmente en los caminos de Francia y España hacia Santiago de Compostela, donde los monjes, al suprimir el pan de centeno y ofrecer el pan de trigo, lograban el "milagro" de curar la isquemia de las extremidades, debida a los efectos de un alcaloide del ergot o cornezuelo de centeno, un hongo ascomiceta, parásito del género claviceps (Claviceps purpúrea) que parasita los granos del cereal (3).

Los derivados del ergot son los compuestos nitrogenados de origen fúngico más abundantes en la naturaleza. Alrededor de 80 derivados del ergot provienen de la especie Claviceps; todos tienen un anillo tetracíclico común denominado ergolina (4). De acuerdo con modificaciones estructurales de este se originan tres tipos: derivados del ácido lisérgico (a este pertenecen la ergometrina, ergovasina, metilergonovina), ergopéptidos (ergotamina, bromocriptina, ergolida) y clavinas (ninguno de este grupo tiene uso médico) (4). Todos estos compuestos tienen mayor o menor efecto de vasoconstricción sistémica, capacidad uterotónica y riesgo potencial de producir ergotismo (5).

La ergonovina (metilergometrina) es un antagonista del receptor de serotonina en varios músculos lisos y en el sistema nervioso central. En los vasos sanguíneos es antagonista débil del receptor de dopamina y agonista parcial del receptor alfa adrenérgico. Sin embargo, su principal efecto es uterotónico, posiblemente por agonismo parcial sobre el receptor de serotonina tipo 2 (5-HT2) (4). Tiene metabolismo hepático mediante el sistema enzimático CYP3A4, por tanto no se debe usar con fármacos que bloqueen este sistema tales como algunos inhibidores de proteasas, macrólidos, quinolonas y antifúngicos azólicos (fluconazol, miconazol e itraconazol), pues su interacción puede favorecer la aparición de ergotismo (4). La bromocriptina es un derivado semisintético de la alfa-ergocriptina, antagonista débil de los receptores de serotonina y alfa-adrenérgico; sin embargo, es un potente agonista de los receptores de dopamina, principalmente el tipo D2, estimula tanto receptores pre como possinápticos, favoreciendo la liberación de dopamina e inhibiendo su recaptación. Por tanto, los efectos farmacológicos son variables y son la sumatoria de sus propiedades agonistas y antagonistas sobre los receptores adrenérgicos, dopaminérgicos y serotoninérgicos (5). Los derivados del ergot que actúen sobre los receptores 5-HT1 y 5-HT2 inducirán vasoconstricción. Existen teorías contradictorias para explicar los efectos sobre el SNC y su fatalidad. Lo cierto es que estas moléculas penetran al sistema nervioso central y sus concentraciones allí son mucho más bajas que en la sangre periférica (por lo que no se produce ergotismo gangrenoso) (1). Algunos derivados del ergot inhiben la glicoproteína $\mathrm{P}$, encargada de extruir ciertas moléculas dañinas para las neuronas, esto ayudaría a explicar por qué se altera la barrera hematoencefálica en estos casos (1). De acuerdo con la pérdida o conservación de la autorregulación cerebral (que depende de factores 
anatómicos, metabólicos y de regulación vascular), se determinará la respuesta clínica (cefalea, isquemia cerebral, hemorragia intracerebral) y el carácter de reversibilidad o no de la condición, independientemente de la presión arterial media (6).

Se presentan dos casos de muerte materna por ergotismo del sistema nervioso central relacionadas con el uso de bromocriptina (caso 1) y metilergometrina (caso 2). Se busca establecer si los fármacos derivados del ergot utilizados en el puerperio se relacionan con eventos neurológicos adversos. Se considera la pertinencia de la publicación dado el amplio uso que tienen estos fármacos en el ejercicio de la obstetricia, tanto para la supresión de la lactancia (uso no aprobado) como en la hemorragia posparto.

\section{REPORTE DE CASOS}

Los casos presentados fueron analizados y discutidos en comités técnicos e interinstitucionales de acuerdo con el Protocolo Nacional de Vigilancia de la Mortalidad Materna. Fue en ellos en los que se consideró que las muertes estaban relacionadas con los medicamentos derivados del ergot.

\section{Caso 1}

Mujer de 38 años, atendida en una institución de tercer nivel de complejidad de la ciudad de Medellín, que recibe población de los regímenes subsidiado y contributivo del sistema de seguridad social en Colombia. Remitida por una institución de menor nivel de complejidad, en su cuarta gestación, con antecedente de un embarazo ectópico y dos cesáreas previas (se desconocen las indicaciones de estas). Tenía historia de cefalea en los 10 años anteriores, diagnosticada como migraña, y tratada de manera sintomática. Sin estudios de neuroimágenes previos. Ingresó normotensa a la visita prenatal en la semana 6 (110/70). En la semana 18 se documentó presión arterial (PA) de 140/80. En la semana 27 de gestación consultó en una institución de baja complejidad, por cefalea de cinco días de evolución; se encontró con PA de 158/100, se inició manejo farmacológico con sulfato de magnesio, alfametildopa y nifedipino, y fue remitida a la institución de tercer nivel con diagnóstico de preeclampsia. Ingresó con PA de 197/127, se continuó sulfato de magnesio y se inició tratamiento con labetalol intravenoso. Los resultados de laboratorio fueron: hemoglobina ( $\mathrm{Hb}):$ 13,8 g/dl; plaquetas: 109000; alanino aminotransferasa (ALT): $123 \mathrm{U} / \mathrm{L}$; aspartato aminotransferasa (AST): $114 \mathrm{U} / \mathrm{L}$; deshidrogenasa láctica (DHL): $1302 \mathrm{U} / \mathrm{L}$; creatinina: $0,8 \mathrm{mg} / \mathrm{dl}$; proteinuria ocasional de 30 $\mathrm{mg} / \mathrm{dl}$. Se documentó además feto con restricción del crecimiento intrauterino. Ante el diagnóstico de síndrome Hellp y dos cesáreas previas, se decidió terminar gestación por cesárea. Se obtuvo feto de $700 \mathrm{~g}$ de peso con signos de asfixia perinatal. En el posoperatorio inmediato, la paciente presentó edema agudo de pulmón y se continuó la vigilancia en la unidad de cuidado intensivo. La hipertensión se controló con metoprolol, clonidina, prazosín y furosemida. En el tercer día del puerperio (PA de $130 / 80$ ) se inició bromocriptina 2,5 mg vía oral cada 8 horas por congestión mamaria). Seis horas después de iniciar la medicación la paciente reinició con cefalea intensa, fotopsias, acompañada de vómito. La presión arterial sistólica osciló entre 160 y $170 \mathrm{~mm} / \mathrm{Hg}$ y la diastólica sobre 90 y $100 \mathrm{~mm}$ / $\mathrm{Hg}$. El origen de la cefalea se interpretó como producto de la anestesia conductiva empleada durante la cesárea. Se suministró manejo sintomático con morfina y metoclopramida, y se aumentó la dosis de antihipertensivos. Se reinició el sulfato de magnesio y se solicitó TAC simple de cráneo. Este fue normal, la cefalea no mejoró y ante su persistencia se decidió realizar punción lumbar. El estudio de líquido cefalorraquídeo fue normal. Para el control de la hipertensión fue necesario reiniciar nitroprusiato de sodio. En el quinto día del puerperio la paciente aún se encontraba con la bromocriptina, presentó monoparesia superior derecha y afasia. Requirió nuevamente soporte ventilatorio dado el deterioro neurológico; se consideró que los signos y síntomas obedecían a vasoespasmo arterial, por 
lo cual se solicitó TAC contrastado de cráneo. El reporte fue: "disminución de la densidad del parénquima cerebral en forma generalizada, cambios por vasoespasmo severo". Se solicitó arteriografía cerebral para aclarar el diagnóstico, la cual evidenció vasoespasmo severo en los segmentos A1 y M1 del lado izquierdo, con compromiso A2 y A3 ipsilateral. No había vasoespasmo en fosa posterior. Se realizó terapia endovascular con nimodipina intraarterial y heparina, con respuesta moderada, sin lograrse resolver del todo el vasoespasmo. A las seis horas del procedimiento presentó midriasis. Se diagnosticó la muerte cerebral. La bromocriptina nunca fue suspendida; no se sospechó posible efecto adverso de esta.

\section{Caso 2}

Mujer de 37 años, en su segunda gestación, atendida en institución de tercer nivel que recibe población de los regímenes subsidiado y contributivo del sistema de seguridad social en Colombia. Tenía antecedente de hipertensión arterial esencial y dislipidemia desde los 22 años, tratada con captopril e hidroclorotiazida, los cuales había suspendido de forma voluntaria cinco meses atrás. Su primera gestación se complicó por preeclampsia grave y terminó en cesárea tres años antes, por estado fetal no satisfactorio. Sin otros antecedentes patológicos de importancia, ni obesidad. Realizó cinco visitas prenatales. En la semana 9 con presión arterial (PA) de 130/80 mm/Hg. La PA en las posteriores osciló entre 110/70 y 120/80 mm/Hg. En la semana 32 se le inició alfametildopa, sin indicación clara por historia clínica, en dosis de $250 \mathrm{mg}$ cada 8 horas. En la semana 40 consultó por cefalea y disminución de movimientos fetales de una semana de evolución. Se encontró una PA de 120/80 y se decidió realizar cesárea por cesárea previa y cérvix no favorable. A los 30 minutos posparto presentó sangrado vaginal abundante, el cual se manejó con la aplicación de 0,2 mg de metilergometrina intramuscular (no hubo aplicación de otros medicamentos para controlar la hemorragia); 25 minutos después de su aplicación presentó una convulsión tónico clónica generalizada con presión arterial de 57/30 y pulso de $44 / \mathrm{min}$. No hubo presencia de síntomas de irritación cortical previos al evento. Se ingresó a la unidad de cuidado intensivo. Mientras se trasladaba al servicio de imagenología para realizarle una tomografía cerebral, presentó paro cardio-respiratorio y falleció cuarenta minutos después de las maniobras de reanimación.

\section{MATERIALES Y MÉTODOS}

Se realizó revisión de la literatura, registrada en la base de datos Medline via PubMed. Los términos empleados para la búsqueda fueron: derivados del ergot, alcaloides del ergot, bromocriptina, angeítis cerebral posparto, ergotismo, enfermedad vascular cerebral posparto, síndromes de vasoconstricción cerebral. Se buscaron, sin límite de tiempo, reportes de caso, reportes de series de caso, revisiones de tema y, en caso de ser pertinente, se revisó la bibliografía citada en los artículos encontrados. Se buscaron informes o alertas de seguridad de agencias reguladoras tales como: la Federal Drugs Administration (FDA), la European Medicines Agency (EMA) y del Instituto Nacional de Vigilancia de Medicamentos y Alimentos (Invima) (Colombia). Se consultaron artículos en inglés, francés y español.

Aspectos éticos. Para la publicación se contó con la revisión y aprobación, tanto de los aspectos éticos como científicos, de la Secretaría seccional de Salud de Antioquia (Comité de salud sexual y reproductiva). Para fines de la publicación y para mantener la reserva de los análisis se omiten las instituciones donde fueron atendidas. La forma en que se presenta la información protege la confidencialidad de las pacientes y las instituciones.

\section{RESULTADOS}

Se identificaron 88 artículos, para la revisión se incluyeron 16 publicaciones que cumplieron con los criterios de búsqueda (7-22), además se verificó que se describiera la relación del medicamento con el evento adverso presentado, tanto en su prescripción como en la mejoría del cuadro al suspenderlo. Por 
otro lado, se verificó que se hubieran descartado otras causas que pudieran explicar el cuadro clínico. De los artículos seleccionados, uno corresponde al reporte de casos producto de la investigación de farmacovigilancia francesa y los restantes a reportes de casos.

Las razones de exclusión fueron: 43 informaron eventos adversos no relacionados con el sistema nervioso central (SNC), 20 por no informar de eventos adversos, 5 corresponden a duplicidades de un mismo autor que publicó en diferentes revistas (solo se tomó una de ellas), 2 por no tener acceso completo (sin embargo, su título sí sugiere evento adverso en SNC) (23, 24), 1 por ser evento adverso por medicamento diferente a derivado del ergot.

Se lograron identificar 33 casos. Del reporte francés, que incluye 105 casos de eventos adversos asociados a bromocriptina, se tienen en cuenta solo los 15 relacionados con efectos sobre el SNC (7), los demás corresponden a eventos isquémicos cardiacos y desórdenes psiquiátricos. Se identificaron dos casos fatales $(8,18)$, uno asociado al uso de metilergonovina intravenosa para el alumbramiento y el otro a ergometrina oral usado como abortivo en la semana 20 de gestación. Tres casos reportan el uso de metilergonovina para realizar alumbramiento durante la cesárea, en dos de ellos se usó de forma venosa. En la tabla 1 se resumen los artículos, el sitio y año de publicación, el medicamento relacionado y otras variables clínicas e imagenológicas.

Independiente del tipo de medicamento utilizado, predominan los hallazgos isquémicos (sugestivos de vasoespasmo cerebral). En tres casos se reportó hemorragia intracerebral, uno de estos fue fatal (18). El síntoma más frecuente de presentación fue la cefalea intensa, seguida de la convulsión. Solo en nueve casos se logró identificar antecedente de hipertensión o preeclampsia, y en cuatro migraña. La gran mayoría de las pacientes fueron sanas. El inicio de los síntomas neurológicos posteriores a la aplicación o ingesta del medicamento fue de menos de 30 minutos cuando se usó ergometrina, y de 6 horas a 19 días cuando se usó bromocriptina.
La indicación para el uso de bromocriptina en todos los casos fue suprimir la lactancia, no se informan las razones para hacerlo. En 1 caso reportado se usó methergolina con este fin (17). La indicación para ablactación de la bromocriptina fue aprobada por la FDA en 1980, pero en 1994 se suspendió su uso para tal fin dado el reporte de 9 casos fatales y de casos de episodios convulsivos asociados (25). Los usos aprobados por la FDA son para la hiperprolactinemia y prolactinomas hipofisiarios (4).

En el sistema de farmacovigilancia colombiano, liderado por Invima, no se encontraron reportes de eventos adversos serios asociados a estos medicamentos. Sin embargo, la subnotificación es un problema reconocido. En Francia fue del $90 \%$, solo un caso de los publicados en la literatura estaba reportado en su sistema (7.)

Los dos casos presentados de mortalidad asociada en este reporte guardan relación temporal con la aplicación de los medicamentos y los síntomas del sistema nervioso central presentados en ambos, y la neuroimagen en uno de ellos sugiere ergotismo del sistema nervioso central. En uno de los casos se sospechó vasoespasmo cerebral, pero no se relacionó con el uso de bromocriptina. En el otro caso, no se sospechó la relación del evento con la aplicación de la metilergometrina. En los comités de análisis donde se presentaron ambos casos se discutió y se consideró esta asociación farmacológica como la causa desencadenante de las muertes. Ambas pacientes tenían factores de riesgo clínico para eventos vasoespásticos del sistema nervioso central.

Se ha sugerido una sensibilidad idiosincrática de los vasos cerebrales a sustancias vasoactivas (14). El puerperio y la presencia de morbilidades como preeclampsia-eclampsia o migraña parecerían predisponer a una mayor respuesta vasoactiva a medicamentos derivados del ergot (12). La angiopatía cerebral posparto, que puede relacionarse o no con medicamentos se puede presentar dentro de los primeros 30 días posparto (26); en este sentido, los casos reportados guardan relación con este criterio. 


\section{CONCLUSIÓN}

Se debe reconocer el ergotismo del sistema nervioso central en el puerperio, por el uso de medicamentos tales como bromocriptina y metilergonovina, como una entidad potencialmente fatal. La bromocriptina, aun en paciente previamente sana, no está indicada para suprimir la lactancia. Se debe evitar el uso intravenoso de la metilergonovina. Es importante crear una cultura de reporte de eventos adversos serios de estos medicamentos en nuestro país.

\section{AGRADECIMIENTOS}

Secretaría Seccional de Salud de Antioquia.

\section{REFERENCIAS}

1. Eadie MJ. Convulsive ergotism: epidemics of the serotonin syndrome? Lancet Neurol. 2003 [visitado 2013 Feb 21];2:429-34. Disponible en: http://www. ncbi.nlm.nih.gov/pubmed/12849122

2. Williams TL, Lukovits TG, Harris BT, Harker Rhodes C. A fatal case of postpartum cerebral angiopathy with literature review. Arch Gynecol Obstet 2007 [visitado 2013 Feb 21];275:67-77. Disponible en: http://www. ncbi.nlm.nih.gov/pubmed/16832640

3. De Costa C. St Anthony's fire and living ligatures: a short history of ergometrine. Lancet. 2002 [visitado 2013 Feb 21];359:1768-70. Disponible en: http:// www.ncbi.nlm.nih.gov/pubmed/12049883

4. Schiff PL. Ergot and its alkaloids. Am J Pharm Educ. 2006 [visitado 2013 Apr 9];70:98. Disponible en: http://www.pubmedcentral.nih.gov/articlerender. fcgi?ar tid=1637017\&tool=pmcentrez\&rendertype $=$ abstract

5. De Groot AN, van Dongen PW, Vree TB, Hekster YA, van Roosmalen J. Ergot alkaloids. Current status and review of clinical pharmacology and therapeutic use compared with other oxytocics in obstetrics and gynaecology. Drugs. 1998 [visitado 2013 Feb 21];56:523-35. Disponible en: http://www.ncbi. nlm. nih.gov/pubmed/9806101

6. Belfort MA, Saade GR, Grunewald C, Dildy GA, Varner MA, Nisell H. Effects of blood pressure on orbital and middle cerebral artery resistances in healthy pregnant women and women with preeclampsia. Am J Obstet Gynecol. 1999;180:601-7.
7. Bernard N, Jantzem H, Becker M, Pecriaux C, BénardLaribière A, Montastruc JL, et al. Severe adverse effects of bromocriptine in lactation inhibition: A pharmacovigilance survey. BJOG. 2015;122:1244-51.

8. Bridger MG, Rodan KS. Ergot poisoning causing death in pregnancy. Br Med J. 1958 [visitado 2013 Feb 21];1:28.Disponible en: http://www. pubmedcentral. nih.gov/articlerender.fcgi?artid $=2026632 \&$ tool $=\mathrm{pm}$ centrez\&rendertype $=$ abstract

9. Abouleish E. Postpartum hypertension and convulsion after oxytocic drugs. Anesth Analg. 1976 [visitado 2013 Feb 21];55:813-5. Disponible en: http:// www. ncbi.nlm.nih.gov/pubmed/1033698

10. Kulig K, Moore LL, Kirk M, Smith D, Stallworth J, Rumack B. Bromocriptine-associated headache: possible life-threatening sympathomimetic interaction. Obstet Gynecol. 1991;78(5 Pt 2):941-3. Disponible en: http://www.ncbi.nlm.nih.gov/pubmed/1923236

11. Barinagarrementeria F, Cantú C, Balderrama J. Postpartum cerebral angiopathy with cerebral infarction due to ergonovine use. Stroke. 1992 [visitado 2013 Feb 21];23:1364-6. Disponible en: http://www.ncbi. nlm.nih.gov/pubmed/1519295

12. Janssens E, Hommel M, Mounier-Vehier F, Leclerc X, Guerin du Masgenet B, Leys D. Postpartum cerebral angiopathy possibly due to bromocriptine therapy. Stroke. 1995 [visitado 2013 Feb 21];26:12830. Disponible en: http://www.ncbi.nlm.nih.gov/ pubmed/7839382

13. Lucas C, Deplanque D, Salhi A, Hachulla E, Doumith S. [Benign angiopathy of the puerperium: a clinicoradiological case associated with ingestión of bromocriptine]. Rev Med Interne. 1996 [visitado 2013 Feb 21];17:839-41. Disponible en: http://www. ncbi.nlm.nih.gov/pubmed/8976978

14. Comabella M, Alvarez-Sabin J, Rovira A, Codina A. Bromocriptine and postpartum cerebral angiopathy: a causal relationship? Neurology. 1996 [visitado 2013 Feb 21];46(6):1754-6. Disponible en: http://www. ncbi.nlm.nih.gov/pubmed/8649587

15. Chartier JP, Bousigue JY, Teisseyre A, Morel C, Delpuech-Formosa F. [Postpartum cerebral angiopathy of iatrogenic origin]. Rev Neurol (Paris). 1997 [visitado 2013 Feb 21];153:212-4. Disponible en: http://www.ncbi.nlm.nih.gov/pubmed/9296138

16. Granier I, Garcia E, Geissler A, Boespflug MD, Durand-Gasselin J. Postpartum cerebral angiopathy 
associated with the administration of sumatriptan and dihydroergotamine--a case report. Intensive Care Med. 1999 [visitado 2013 Feb 21];25(5):532-

4. Disponible en: http://www.ncbi.nlm.nih.gov/ pubmed/10401952

17. Crippa G, Sverzellati E, Pancotti D, Carrara GC. Severe postpartum hypertension and reversible cerebral angiopathy associated with ergot derivative (methergoline) administration. Ann Ital Med Int. 2000 [visitado 2013 Apr 9];15:303-5. Disponible en: http:// www.ncbi.nlm.nih.gov/pubmed/11202634

18. Kirsch C, Iffy L, Zito GE, McArdle JJ. The role of hypertension in bromocriptine-related puerperal intracranial hemorrhage. Neuroradiology. 2001 [visitado 2013 Feb 21];43:302-4. Disponible en: http://www.ncbi.nlm.nih.gov/pubmed/11338413

19. Burckard E, Patrigeon RG, Felten D, Combourieu E, Escarment J. [Convulsions due to a postpartum cerebral angiopathy associated with the administration of bromocriptine]. Ann Fr Anesth Reanim. 2003 [visitado 2013 Feb 21];22:46-9. Disponible en: http:// www.ncbi.nlm.nih.gov/pubmed/12738020

20. Barroso B, Bigou P, Martin K, Lippa A, Chabouni L, Dakar A, et al. [Reversible postpartum cerebral angiopathy associated with bromocriptine therapy]. Rev Neurol (Paris). 2004 [visitado 2013 Feb 21];160):1191-3. Disponible en: http://www.ncbi. nlm.nih.gov/pubmed/15602366
21. Ishibashi T, Ishibashi S, Uchida T, Nakazawa K, Makita $K$. Reversible cerebral vasoconstriction syndrome with limb myoclonus following intravenous administration of methylergometrine. J Anesth. 2011 [visitado 2013 Feb 21];25:405-8. Disponible en: http://www.ncbi. nlm.nih.gov/pubmed/21431624

22. Farissier F, Reynaud A, Varvat J, Coudrot M, Garnier P, Tardy B. [Postpartum reversible cerebral angiopathy: an unusual cause of headache]. Ann Fr Anesth Reanim. 2011 [visitado 2013 Feb 21];30:61-3. Disponible en: http://www.ncbi.nlm.nih.gov/pubmed/21185686

23. Gittelman DK. Bromocriptine associated with postpartum hypertension, seizures, and pituitary hemorrhage. Gen Hosp Psychiatry. 1991;13:278-80.

24. Katz M, Kroll D, Pak I, Osimoni A, Hirsch M. Puerperal hypertension, stroke, and seizures after suppression of lactation with bromocriptine. Obstet Gynecol. 1985;66(6):822-4. Disponible en: http:// www.ncbi.nlm.nih.gov/pubmed/3840873

25. De Jong-van den Berg L, Mintzes B. Bromocriptine and lactation suppression: Are the risks acceptable? Pharm World Sci. 1995;17:93-5.

26. Iffy L, Lindenthal JJ, McArdle JJ, McNamara RE, Szodi Z, Ganesh V. Ergotism: a possible etiology for puerperal psychosis. Obstet Gynecol. 1989 [visitado 2013 Feb 21];73(3 Pt 2):475-7. Disponible en: http:// www.ncbi.nlm.nih.gov/pubmed/2915878 


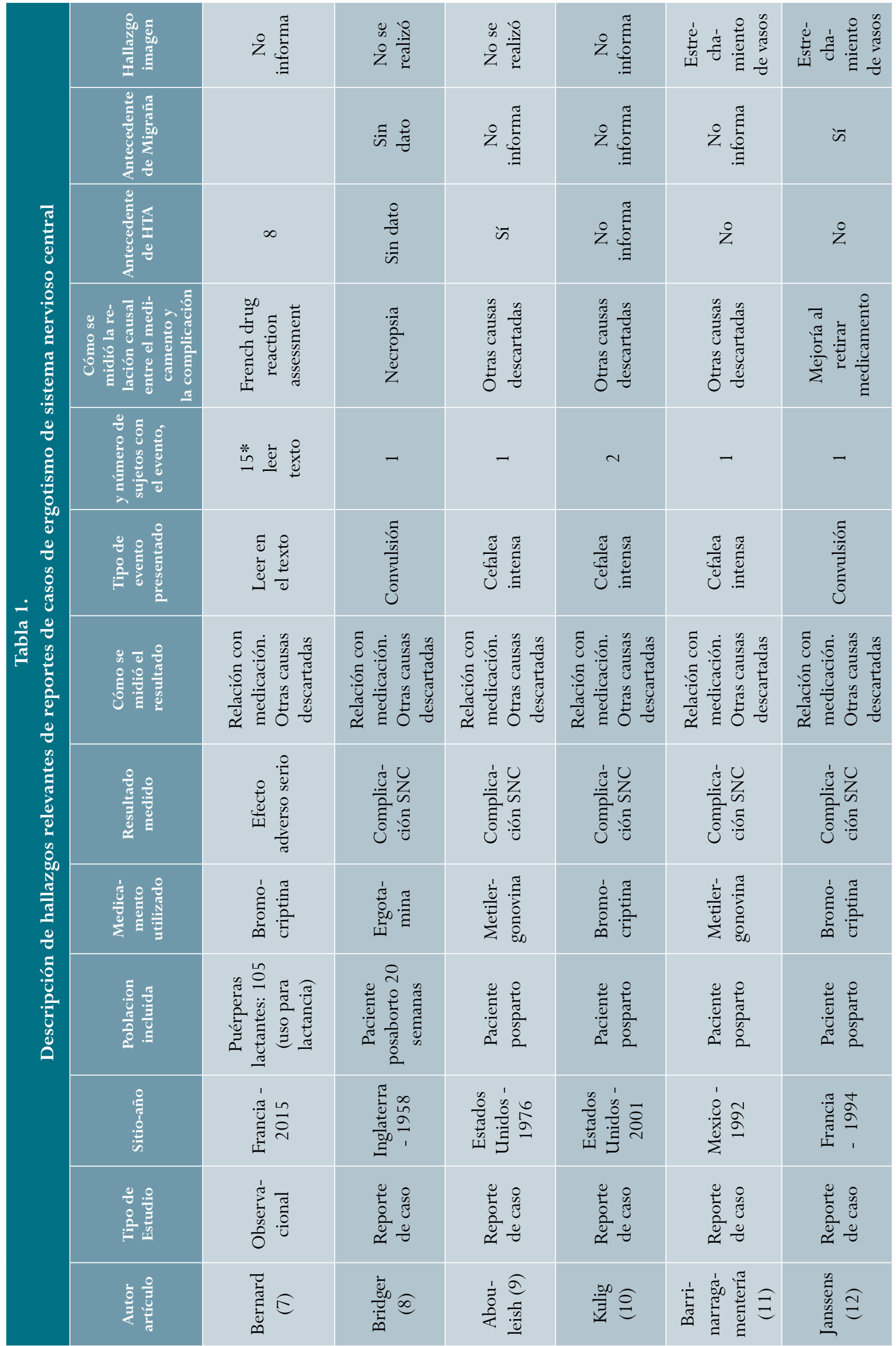




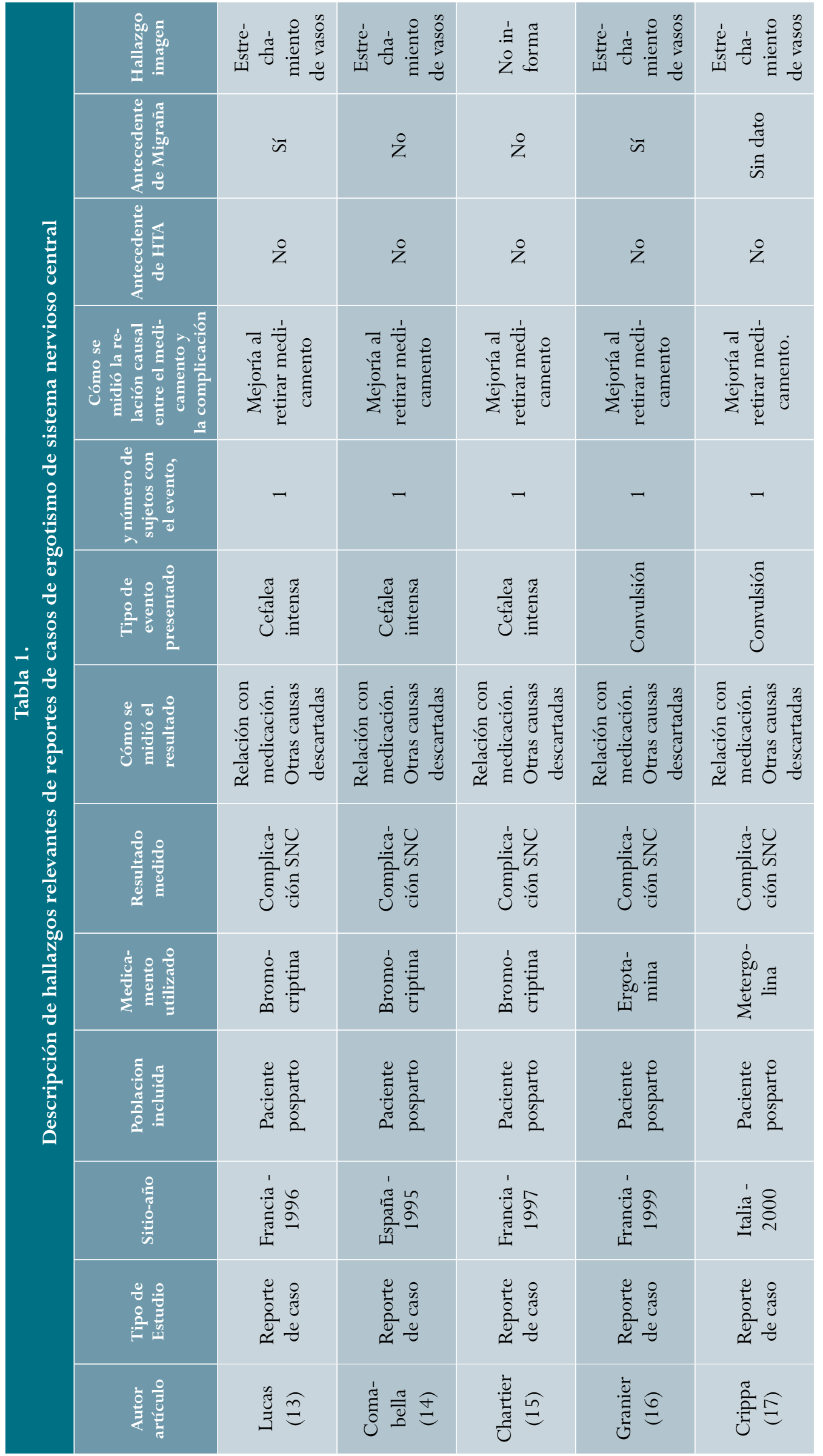




\begin{tabular}{|c|c|c|c|c|c|c|}
\hline & 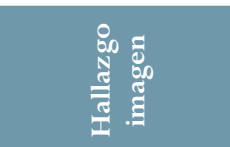 & 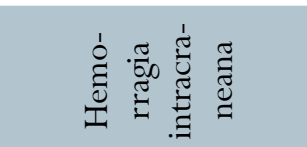 & 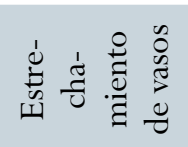 & 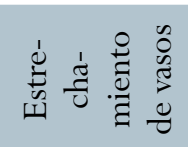 & 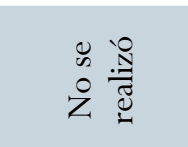 & 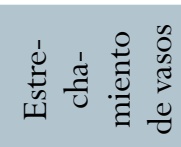 \\
\hline & 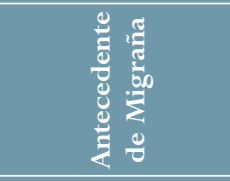 & ż & is & $\stackrel{0}{z}$ & $\ddot{z}$ & $\stackrel{0}{z}$ \\
\hline $\begin{array}{l}\text { हैं } \\
8 \\
0 \\
0\end{array}$ & 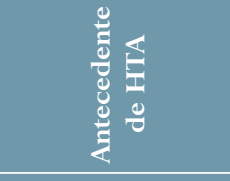 & $\stackrel{\circ}{z}$ & 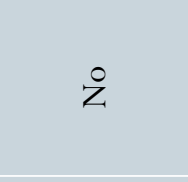 & $\stackrel{\circ}{Z}$ & Z & 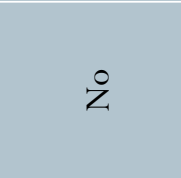 \\
\hline 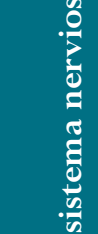 & 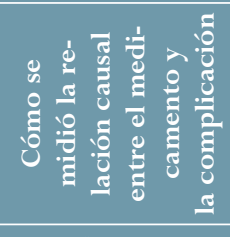 & 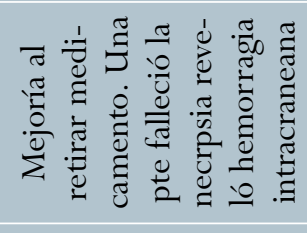 & 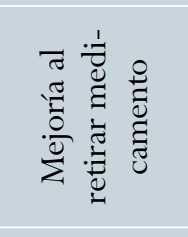 & 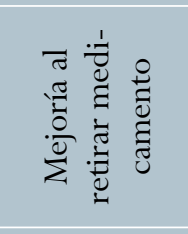 & 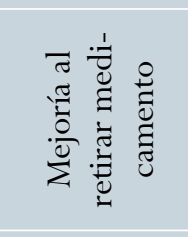 & 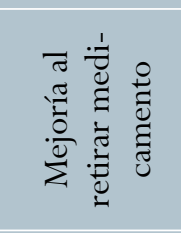 \\
\hline 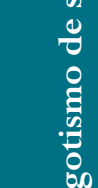 & 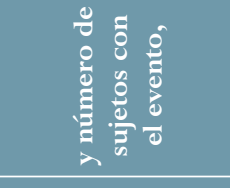 & 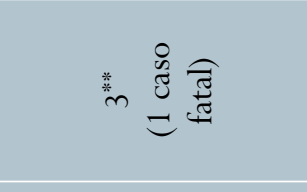 & - & - & - & - \\
\hline 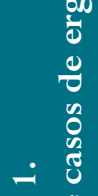 & 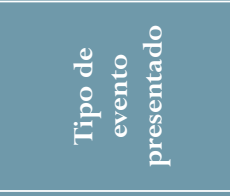 & 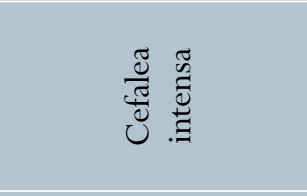 & $\begin{array}{l}\frac{10}{0} \\
\frac{0}{3} \\
0 \\
0\end{array}$ & $\frac{\sqrt[0]{0}}{\frac{0}{0}}$ & 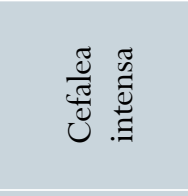 & 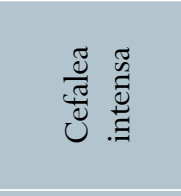 \\
\hline 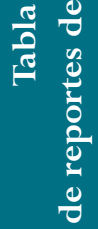 & 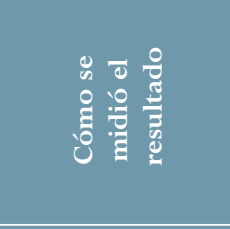 & 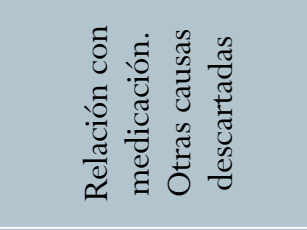 & 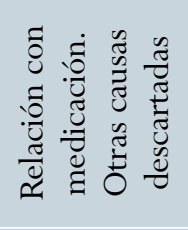 & 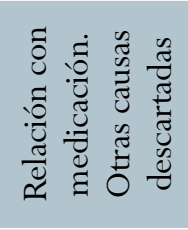 & 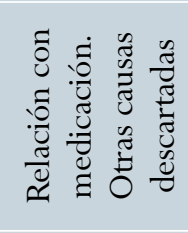 & 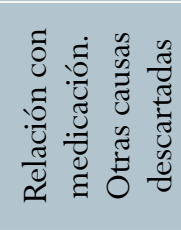 \\
\hline$\frac{8}{0}$ & 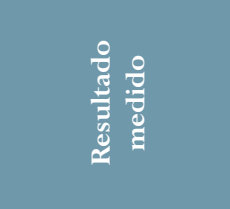 & 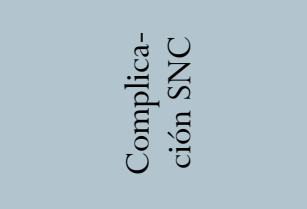 & 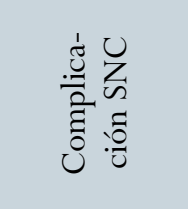 & 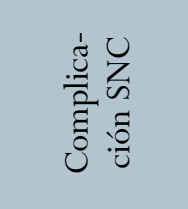 & 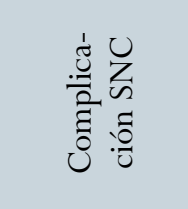 & 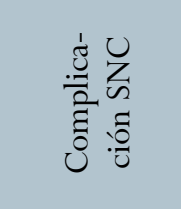 \\
\hline$\frac{\sqrt{8}}{\frac{\pi}{8}}$ & 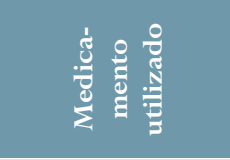 & 竞: & 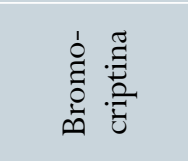 & 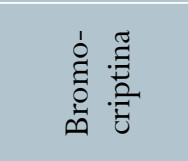 & 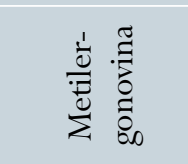 & 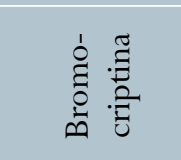 \\
\hline 递 & $\begin{array}{l}\frac{5}{0} \\
\frac{0}{0} \\
\frac{0}{0} \\
\frac{0}{0} \\
0\end{array}$ & 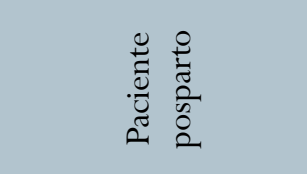 & 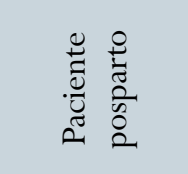 & 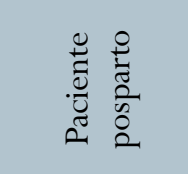 & 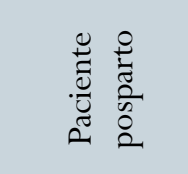 & 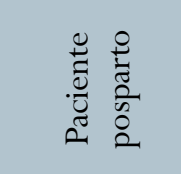 \\
\hline & 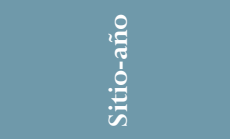 & 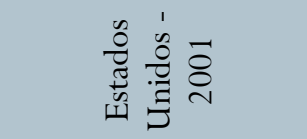 & 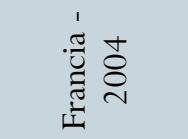 & 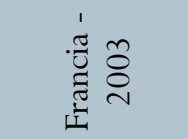 & 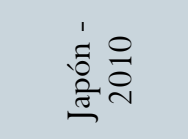 & 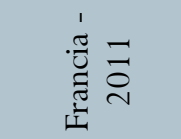 \\
\hline & 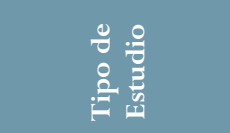 & 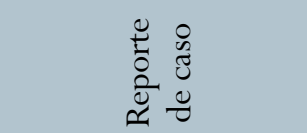 & 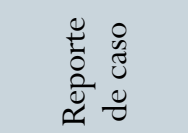 & 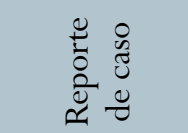 & 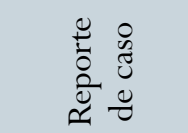 & 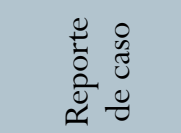 \\
\hline & 总莚 & 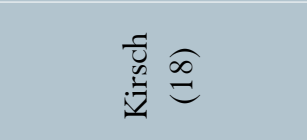 & 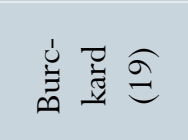 & 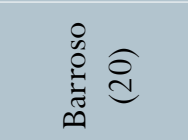 & 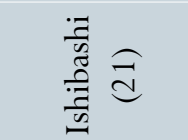 & 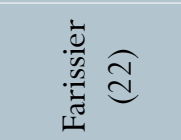 \\
\hline
\end{tabular}

